\title{
Understanding COVID-19-related Burnout in Qatar's Community Pharmacists using the Job Demands-Resources Theory
}

\section{Hiba Mohammed, Shahd Elamin, Dr Maguy El-Hajj, and Dr Alla El-Awaisi College of Pharmacy, QU health, Qatar University}

\section{Background}

Community pharmacists are one of the most accessible front-liners against the COVID-19 pandemic

- Community pharmacists are at risk of burnout, a psychological syndrome that occurs due to prolonged exposure to stressors

The literature exploring the factors that lead to burnout in community pharmacists during the COVID-19 pandemic is lacking

- The Jobs Demands-Resource (JD-R) model examines the interaction between job-related and personal-related demands and resources

It then relates these factors to the subsequential outcomes of burnout and work engagement

\section{Study objectives}

To understand the factors that contribute to community pharmacists' burnout as explained by the JD-R Theory

- To identify currently used strategies by community pharmacists to mitigate burnout

- To make recommendations about interventions that could reduce burnout in community pharmacists during COVID-19

\section{Methods}

Qualitative study using focus groups

- Phenomenological methodology

- Focus group guide based on the JD-R Model

Population of interest: Community pharmacists

Language: English

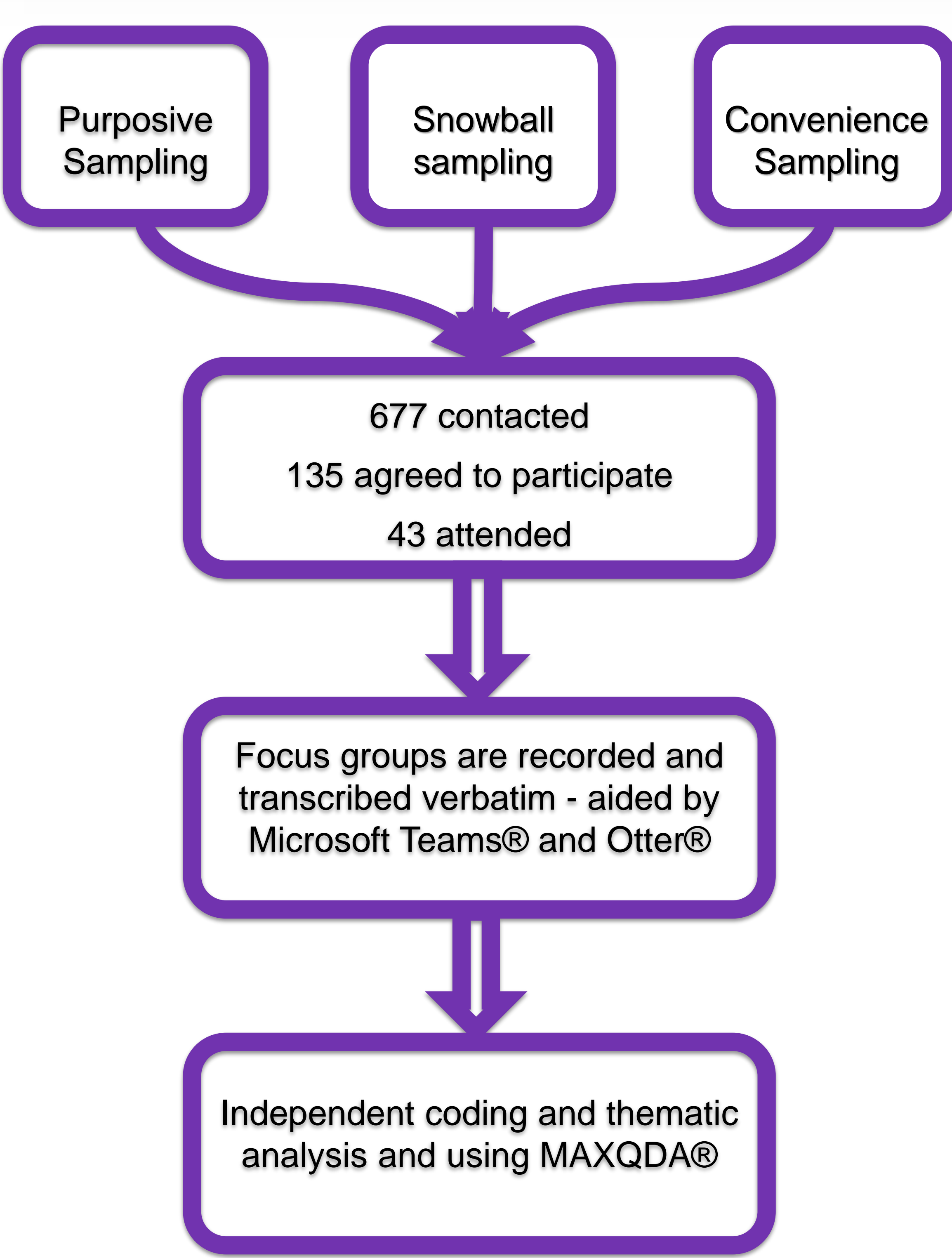

Figure 1: Schematic representation of the methods

\section{Results}

- Based on findings of 6 focus groups, 6 dyadic and mini-focus groups, and 4 individual interviews were conducted, the following themes emerged

\section{Demands/Resources Intersection}

- Financial status and job security

- Quality of patient interaction

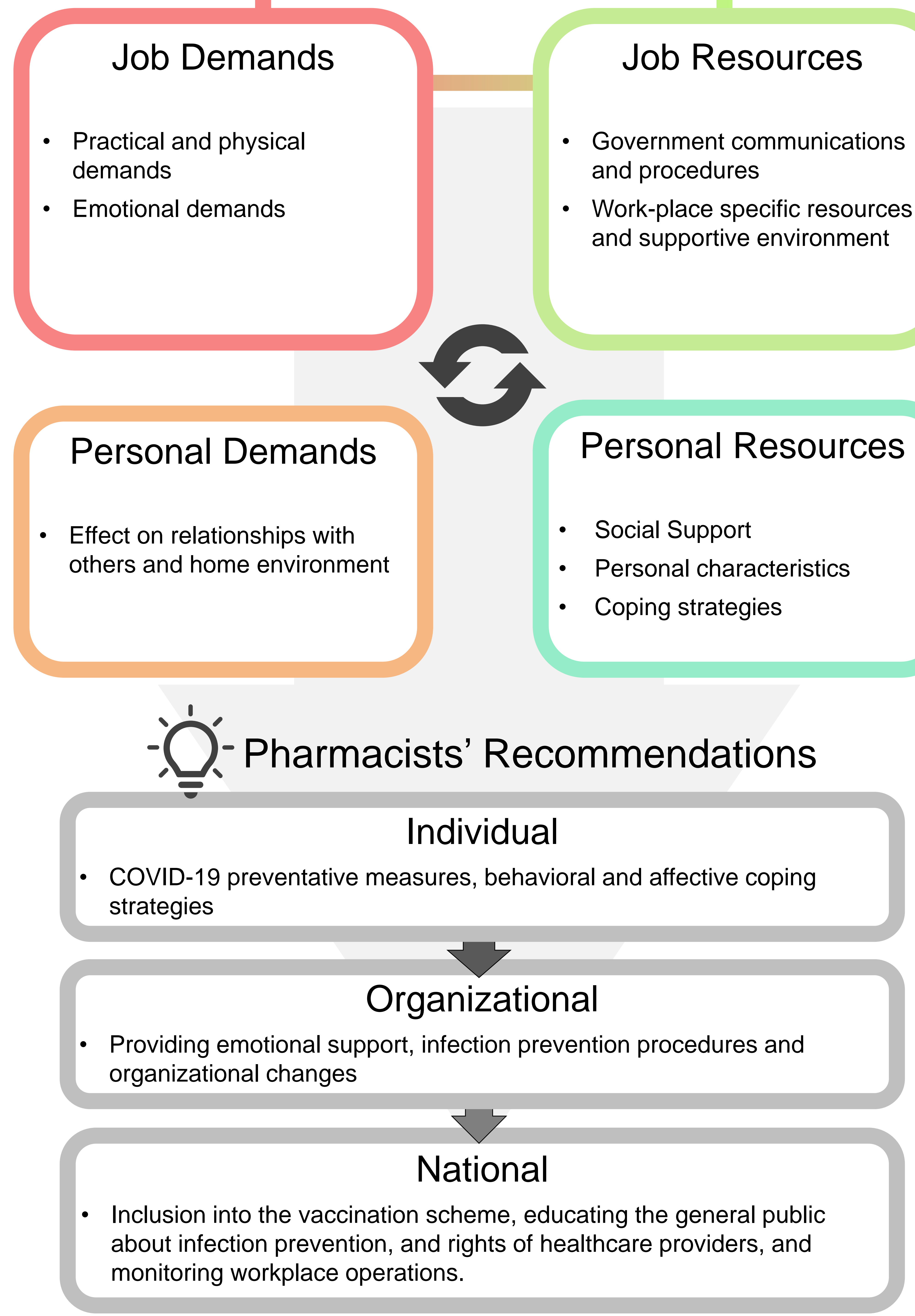

\section{Conclusion}

- To our knowledge, this is the first study to examine the factors that influence burnout in community pharmacists during the COVID-19 pandemic

- The practical and emotional demands identified are a call for policymakers to make specific interventions that improve pharmacists' wellbeing by:

$>$ Reducing workload through task allocation and adequate staffing

Raising awareness in patients on improving interactions

Mental health, counselling and self-help resources

- Future research is needed to understand the correlations between these factors and burnout

- The long-term mental health consequences in community pharmacists must also be identified 